\title{
IDENTIFIABILITY OF AN UNKNOWN COEFFICIENT IN A NONLINEAR DIFFUSION EQUATION*
}

By

\section{CHOULLI}

Laboratoire d'Automatique et d'Analyse des Systèmes du CNRS, Toulouse, France

\begin{abstract}
We consider the problem of identifying an unknown coefficient in a onedimensional diffusion equation from overspecified data measured in the interior of the domain. We obtain an identifiability result which is used to derive a uniqueness result for the inverse problem.
\end{abstract}

1. Introduction. Let us consider the nonlinear diffusion problem given by:

$$
\begin{cases}L[u]=0, & \text { in } \bar{Q}_{T}=[0,1] \times[0, T] \text { for } T>0 \\ u(x, 0)=0, & 0 \leq x \leq 1 \\ u(0, t)=f_{0}(t), & 0 \leq t \leq T \\ u(1, t)=f_{1}(t), & 0 \leq t \leq T\end{cases}
$$

where $L$ is the operator:

$$
L=a(\cdot) \partial_{x x} \cdot-\partial_{t} \cdot
$$

We define, for some fixed $x_{0}$ in $] 0,1[$ :

$$
\begin{aligned}
C: U & \rightarrow U_{0} \\
u & \mapsto u\left(x_{0}, \cdot\right)=h
\end{aligned}
$$

and

$$
\begin{aligned}
A: \mathscr{A} & \rightarrow U \\
a & \mapsto u
\end{aligned}
$$

where $U$ is the space of smooth solutions of (1.1) for given $a$ in the class of admissible coefficients $\mathscr{A}$ and $U_{0}$ is the space of overspecified data $h$. The set of admissible coefficients $\mathscr{A}$ is given in the fourth section.

In this paper we study the mapping $C \circ A$ which carries the unknown coefficient into the overspecified data $h$. We prove for some class of given data $f_{0}$ and $f_{1}$ that the mapping $C \circ A$ is injective (identifiability). This result can then be used to conclude that a solution of the inverse problem, assuming it exists, must be unique. In the inverse problem we want to recover the coefficient $a$ in problem (1.1) from the additional condition $u\left(x_{0}, \cdot\right)=h$.

*Received May 22, 1987. 
Since the technique used to derive the injectivity of the mapping $C \circ A$ relies on the maximum principle for linear parabolic equations, we need to introduce some results.

Statement of the maximum principle. ([6], Chapter 3). Let $C^{2,1}$ denote the usual space of functions $u$ continuous in $\bar{Q}_{T}$ having continuous derivatives $\partial_{x} u, \partial_{x x} u, \partial_{t} u$ in $\bar{Q}_{T}$. Let $\alpha$ be defined and bounded in $\bar{Q}_{T}, \alpha_{0} \leq \alpha(x, t)$ in $\bar{Q}_{T}$, where $\alpha_{0}$ is some positive constant, and

$$
P=\alpha(x, t) \partial_{x x} \cdot-\partial_{t} \cdot
$$

THEOREM 1.1. Let $P$ be defined by (1.2) and let $z=z(x, t) \in C^{2,1}\left(\bar{Q}_{T}\right)$ satisfy $P[z] \geq 0$ in $\bar{Q}_{T}$. If $z$ is not constant in $\bar{Q}_{T}$ then the maximum of $z$ is attained in $\Gamma=\partial \bar{Q}_{T} \backslash\{(x, t), 0<x<1, t=T\}$.

Corollary 1.1. Let $z=z(x, t) \in C^{2,1}\left(\bar{Q}_{T}\right)$ satisfy $P[z]+\beta z \geq 0$ in $\bar{Q}_{T}$, where $P$ is defined by (1.2) and $\beta$ is a bounded function in $\bar{Q}_{T}$. Then $z \leq 0$ in $\Gamma$ implies $z \leq 0$ in $\bar{Q}_{T}$.

2. Solution of the nonlinear equation. In this section we study the existence and uniqueness of the nonlinear diffusion equation (1.1). We prove under certain conditions on $a, f_{0}$, and $f_{1}$ that this solution is at least in $C^{2,1}\left(\bar{Q}_{T}\right)$.

We suppose first that

$$
\delta \leq a(s) \leq \mu \text { for each } s \text { in } I=[0, M],
$$

where $\delta, \mu$ are some constants, $0<\delta<\mu, M=\max _{Q_{T}} u_{\mu}(x, t)$, and $u_{\mu}=u_{\mu}(x, t)$ denotes the solution of $(1.1)$ in the case that the coefficient $a$ is identically equal to $\mu$ and $f_{0}, f_{1} \in D_{3,2}=\left\{h \in C^{3}[0, T], h(0)=0, h^{\prime} \in D_{2}\right\}$ with $D_{2}=\left\{h \in C^{2}[0, T]\right.$, $\left.h(0)=h^{\prime}(0), h^{\prime}(t)>0,0<t \leq T\right\}$.

Proposition 2.1. If $a$ satisfies $(2.1), f_{0}, f_{1} \in D_{3,2}$ and $u=u(x, t) \in C^{2,1}\left(\bar{Q}_{T}\right)$ satisfies (1.1), then

$$
0 \leq u_{\delta}(x, t) \leq u(x, t) \leq u_{\mu}(x, t) .
$$

In addition, $0 \leq u(x, t) \leq M$ in $\bar{Q}_{T}$. Here, $u_{\delta}$ (resp., $u_{\mu}$ ) denotes the solution of (1.1) in the case that $a=\delta$ (resp., $a=\mu$ ).

Proof. Let $v_{k}=\partial_{t} u_{k}, k=\delta, \mu$. Then $v_{k}$ satisfies:

$$
\begin{cases}\partial_{t} v_{k}=k \partial_{x x} v_{k}, & \text { in } \bar{Q}_{T}, \\ v_{k}(x, 0)=0, & 0 \leq x \leq 1, \\ v_{k}(\cdot, t)=f_{0}^{\prime}(t), & 0 \leq t \leq T, \\ v_{k}(1, t)=f_{1}^{\prime}(t), & 0 \leq t \leq T .\end{cases}
$$

$v_{k}$ is in $C^{2,1}\left(\bar{Q}_{T}\right)$ [4]. By application of Theorem 1.1 to $v_{k}, k=\delta, \mu$, we deduce that $\partial_{x x} u_{k}=\frac{1}{k} \partial_{t} u_{k}=\frac{1}{k} v_{k} \geq 0$ in $\bar{Q}_{T}$. It is clear from Theorem 1.1 applied to $u_{\delta}$ that $u_{\delta} \geq 0$ in $\bar{Q}_{T}$. Now, if we let $w_{k}=u-u_{k}$ in $Q_{T}$, then $w_{k}$ satisfies:

$$
\begin{array}{r}
(a \circ u)(x, t) \partial_{x x} w_{k}-\partial_{t} w_{k}=-[(a \circ u)(x, t)-k] \partial_{x x} u_{k} \quad \text { in } \bar{Q}_{T}, \\
\text { and } w_{k}=0 \text { in } \Gamma, \quad k=\delta, \mu .
\end{array}
$$

Finally, we obtain (2.2) by application of Theorem 1.1 to $w_{k}, k=\delta, \mu$. 
Lemma 2.1 [5]. If $a \in C^{2}(I)$ satisfies (2.1) and $f_{0}, f_{1} \in D_{3,2}$ then the problem (1.1) has a unique solution $u=u(x, t)$ at least in $C^{2,1}\left(\bar{Q}_{T}\right)$.

3. Properties of $u$ and $\partial_{x x} u$. In analysing the identifiability problem, it will be necessary to have certain information regarding the solution $u$ of the problem (1.1) and its derivative $\partial_{x x} u$. For the purpose of extracting this information, we consider the following problem:

$$
\begin{cases}P[z]=0, & \text { in } \bar{Q}_{T}, \\ z(x, 0)=0, & 0 \leq x \leq 1, \\ z(0, t)=g_{0}(t), & 0 \leq t \leq T, \\ z(1, t)=g_{1}(t), & 0 \leq t \leq T,\end{cases}
$$

where $P$ is the linear operator defined by (1.2).

For $\alpha \in C^{1}\left(Q_{T}\right), g_{0}, g_{1} \in D_{2}$, the problem (3.1) has a unique solution in $C^{2,1}\left(\bar{Q}_{T}\right)$ [4].

Proposition 3.1. Let $z=z(x, t)$ be the solution of the problem (3.1). Then for each $t_{0}, 0<t_{0}<T$, there exists a constant $K=K\left(t_{0}\right)>0$ such that $z(x, t) \geq K$, $0 \leq x \leq 1, t_{0} \leq t \leq T$ with $\lim _{t_{0} \rightarrow 0} K\left(t_{0}\right)=0$.

Proof. For $\theta>0$, let $M(x, t)=\frac{1}{\sqrt{\theta t}} \exp \left(\frac{-(x+1)^{2}}{4 \theta t}\right), 0 \leq x \leq 1,0<t \leq T$ and note that $M(x, 0)=\partial_{x} M(x, 0)=\partial_{x x} M(x, 0)=\partial_{t} M(x, 0)=0,0 \leq x \leq 1$. Then, $M$ satisfies $\partial_{t} M(x, t)=\theta \partial_{x x} M(x, t)$ in $\bar{Q}_{T}$ and

$$
w(x, t)=(1-x) \int_{0}^{t} M(x, t-\tau) g_{0}(\tau) d \tau+x \int_{0}^{t} M(1-x, t-\tau) g_{1}(\tau) d \tau
$$

is the solution of the problem:

$$
\begin{cases}\theta \partial_{x x} w=F(x, t)+\partial_{t} w, & \text { in } \bar{Q}_{T}, \\ w(x, 0)=0, & 0 \leq x \leq 1, \\ w(0, t)=\left.\int_{0}^{t} M(x, t-\tau) g_{0}(\tau) d \tau\right|_{x=0}, & 0 \leq t \leq T \\ w(1, t)=\left.\int_{0}^{t} M(1-x, t-\tau) g_{1}(\tau) d \tau\right|_{x=1}, & 0 \leq t \leq T\end{cases}
$$

where $F(x, t)=-2 \theta \int_{0}^{t} \partial_{x} M(x, t-\tau) g_{0}(\tau) d \tau+2 \theta \int_{0}^{t} \partial_{x} M(1-x, t-\tau) g_{1}(\tau) d \tau \geq 0$ in $\bar{Q}_{T}$. Integrating by parts the expression of $\partial_{x x} w$ we have:

$\partial_{x x} w=\frac{1}{\theta} F(x, t)+\frac{1}{\theta}(1-x) \int_{0}^{t} M(x, t-\tau) g_{0}^{\prime}(\tau) d \tau+\frac{1}{\theta} x \int_{0}^{t} M(1-x, t-\tau) g_{1}^{\prime}(\tau) d \tau$.

Hence, $\partial_{x x} w(x, t) \geq 0$ in $\bar{Q}_{T}$. Now, we choose the constant $\theta$ as follows:

$$
\theta \leq \alpha_{0}, \quad \theta T \leq \frac{1}{\sqrt{2}}, \quad \text { and } \quad G(\theta T) \leq \frac{1}{T}
$$

where $G(X)=\frac{1}{\sqrt{X}} \exp \left(-\frac{1}{4 X}\right)$. Under the stated condition on $\theta$ we have:

$$
w(0, t) \leq g_{0}(t) \text { and } w(1, t) \leq g_{1}(t), \quad 0 \leq t \leq T .
$$

The application of Theorem 1.1 to the difference $(w-z)$ leads to the result $w \geq z$ in $\bar{Q}_{T}$. Finally, we have:

$$
z(x, t) \geq K_{0}(1-x)+K_{1} x, \quad 0 \leq x \leq 1, \quad t_{0} \leq t \leq T,
$$


with

$$
K_{i}=\operatorname{Min}_{t_{0} / 2 \leq t \leq t_{0}} M(1, t) \int_{0}^{t_{0} / 2} g_{1}(\tau) d \tau, \quad i=0,1 .
$$

Thus, the proof is achieved for $K=\operatorname{Min}\left(K_{0}, K_{1}\right)$.

Proposition 3.2. Let $a \in C^{2}(I)$ satisfy (2.1), $f_{0}, f_{1} \in D_{3,2}$ and let $u=u(x, t)$ be the solution of (1.1). Then, for each $s_{1}>0$, there exists a $t_{1}, 0<t_{1} \leq T$, such that $u(x, t) \leq s_{1}, 0 \leq x \leq 1,0 \leq t \leq t_{1}$. In addition, there exists a $t_{0}, 0<t_{0}<t_{1}$, and $s_{0}=s_{0}\left(t_{0}\right), 0<s_{0}<s_{1}$, such that $s_{0} \leq u(x, t) \leq s_{1}, 0 \leq x \leq 1, t_{0} \leq t \leq t_{1}$.

Proof. First, we apply Theorem 1.1 to get $u(x, t) \leq \max \left(f_{0}(t), f_{1}(t)\right)$ in $\bar{Q}_{T}$. The hypothesis on $f_{0}, f_{1}$ implies the existence of $t_{1}, 0<t_{1} \leq T$, satisfying $\max \left(f_{0}\left(t_{1}\right), f_{1}\left(t_{1}\right)\right)$ $\leq s_{1}$. Now, if we choose $t_{0}, 0<t_{0}<t_{1}$, such that $K\left(t_{0}\right)<s_{1}$, where $K\left(t_{0}\right)$ is the constant from Proposition 3.1 for the case of $\alpha=\delta$, then $u_{\delta}(x, t) \geq K\left(t_{0}\right), 0 \leq x \leq 1$, $t_{0} \leq t \leq T$, and the proof is completed by applying Proposition 3.1 with $s_{0}=K\left(t_{0}\right)$.

Proposition 3.3. If $a \in C^{2}(I)$ satisfies (2.1) with $a^{\prime} \geq 0, f_{0}, f_{1} \in D_{3,2}$, and $u=u(x, t)$ is the solution of $(1.1)$, then

1) $\partial_{x x} u(x, t) \geq 0$ in $\bar{Q}_{T}$, and

2) for each $t_{0}, 0<t_{0}<T$, there is a constant $C>0$ such that $\partial_{x x} u(x, t) \geq C$, $0 \leq x \leq 1, t_{0} \leq t \leq T$.

Proof. Let

$$
\begin{aligned}
v & =\partial_{t} u \text { in } \bar{Q}_{T}, \\
L & =(a \circ u)(x, t) \partial_{x x} \cdot-\partial_{t},
\end{aligned}
$$

and

$$
F=\left(a^{\prime} \circ u\right)(x, t) \cdot(a \circ u)(x, t) \cdot\left[\partial_{x x} u(x, t)\right]^{2} \text { in } \bar{Q}_{T} .
$$

Then $v=v(x, t)$ is the solution of the following problem:

$$
\begin{cases}L[v]=-F(x, t), & \text { in } \bar{Q}_{T}, \\ v(x, 0)=0, & 0 \leq x \leq 1, \\ v(0, t)=f_{0}^{\prime}(t), & 0 \leq t \leq T, \\ v(1, t)=f_{1}^{\prime}(t), & 0 \leq t \leq T .\end{cases}
$$

Now, let $\tilde{v}=\tilde{v}(x, t)$ be the solution of the problem:

$$
\begin{cases}L[\tilde{v}]=0, & \text { in } \bar{Q}_{T}, \\ \tilde{v}(x, 0)=0, & 0 \leq x \leq 1, \\ \tilde{v}(0, t)=f_{0}^{\prime}(t), & 0 \leq t \leq T, \\ \tilde{v}(1, t)=f_{1}^{\prime}(t), & 0 \leq t \leq T .\end{cases}
$$

From [4], $v$ and $\tilde{v}$ are at least in $C^{2,1}\left(\bar{Q}_{T}\right)$, and Theorem 1.1 applied to the difference $(v-\tilde{v})$ leads to the result $v-\tilde{v} \geq 0$ in $\bar{Q}_{T}$. By Proposition 3.1, for each $t_{0}, 0<t_{0}<T$, there exists a constant $K>0$ such that:

$$
\tilde{v}(x, t) \geq K, \quad 0 \leq x \leq 1, \quad t_{0} \leq t \leq T .
$$

But

$$
\partial_{x x} u(x, t) \geq \frac{\partial_{t} u(x, t)}{\mu}=\frac{v(x, t)}{\mu} \geq \frac{\tilde{v}(x, t)}{\mu} \text { in } \bar{Q}_{T},
$$

and the proof follows with $C=K / \mu$. 
4. Identifiability. To analyse the mapping $C \circ A$ which carries the unknown coefficient into the overspecified data, we need the definition of the class of admissible coefficients.

Let $\Lambda=\left\{a \in C^{2}\right.$, satisfying $(2.1)$ and $\left.a^{\prime} \geq 0\right\}$. Then $\mathscr{A}$ is said to be a class of admissible coefficients iff $\mathscr{A} \subset \Lambda$ and for each $a, b$ in $\mathscr{A}$ either $a(0) \neq b(0)$ or $a(0)=b(0)$ and $a^{\prime}(0) \neq b^{\prime}(0)$.

We can also choose $\mathscr{A}=\{a \in \Lambda$ and $a$ analytic in $\dot{I}\}$.

TheOREM 4.1. If $a \in \mathscr{A}, f_{0}, f_{1} \in D_{3,2}, u=u(x, t)$ is the solution of (1.1), and $\left.x^{*} \in\right] 0,1[$, then the mapping:

$$
\begin{aligned}
\mathscr{A} & \rightarrow C^{1}[0, T] \\
a & \mapsto u\left(x^{*}, t\right)=h
\end{aligned}
$$

is injective.

The proof of Theorem 4.1 is based on the following proposition:

Proposition 4.1. Let $z=z(x, t) \in C^{2,1}\left(\bar{Q}_{T}\right)$ satisfy the equations:

$$
P[z]=-F(x, t) \text { in } \bar{Q}_{T} \text { and } z=0 \text { in } \Gamma,
$$

where $P$ is defined by (1.2) and $F$ is some function defined in $\bar{Q}_{T}$ having the properties:

1) $F(x, t) \geq 0$ in $\bar{Q}_{T}$, and

2) for each $t_{0}, x_{0}, x_{1}, 0<t_{0}<T$ and $0 \leq x_{0}<x_{1} \leq 1$, there is a constant $C_{0}$ such that $F(x, t) \geq C_{0}, x_{0} \leq x \leq x_{1}, t_{0} \leq t \leq t_{1}$. Then

i) $z(x, t) \geq 0$ in $\bar{Q}_{T}$, and

ii) for each $x^{*}, t_{1}, 0<x^{*}<1$ and $0<t_{1}<T$, there is a constant $C_{1}$ such that $z\left(x^{*}, t\right) \geq C_{1}, t_{1} \leq t \leq T$.

Proof. For i) we apply Theorem 1.1. For ii), let $x^{*}$ satisfy $0<x^{*}<1$ and $t_{1}$ satisfy $0<t_{1}<T$. We choose $t_{0}, x_{0}$, and $x_{1}$ as follows:

$$
0 \leq x_{0}<x^{*}<x_{1} \leq 1 \text { and } 0<t_{0}<t_{1} \text {. }
$$

For $U=\left\{(x, t), x_{0}<x<x_{1}, t_{0}<t<T\right\}$ and $\phi(x, t)=z(x, t)-\sigma\left(t-t_{0}\right)\left(x_{1}-x\right)\left(x-x_{0}\right)$ in $\bar{U}$, we have:

$$
\begin{array}{ll}
\phi\left(x, t_{0}\right)=z\left(x, t_{0}\right) \geq 0, & 0 \leq x \leq x_{1}, \\
\phi\left(x_{0}, t\right)=z\left(x_{0}, t\right) \geq 0, & t_{0} \leq t \leq T, \\
\phi\left(x_{1}, t\right)=z\left(x_{1}, t\right) \geq 0, & t_{0} \leq t \leq T,
\end{array}
$$

and $P[\phi]=P[z]+\sigma\left[\left(x_{1}-x\right)\left(x-x_{0}\right)+2 \alpha(x, t)\left(t-t_{0}\right)\right]$. The hypothesis on $F$ implies

$$
L[\phi] \leq-C_{0}+\sigma\left[\frac{1}{4}\left(x_{1}-x_{0}\right)^{2}+2\left(T-t_{0}\right) \max _{Q_{T}} \alpha(x, t)\right] .
$$

If we let

$$
\sigma=C_{0}\left[\frac{1}{4}\left(x_{1}-x_{0}\right)^{2}+2\left(T-t_{0}\right) \max _{Q_{T}} \alpha(x, t)\right]^{-1}
$$

then $P[\phi] \leq 0$ in $U$. 
The conclusion of Theorem 1.1 is still valid if we change $\bar{Q}_{T}$ by $\bar{U}$. Thus, it follows that $\phi \geq 0$ in $\bar{U}$ and

$$
z(x, t) \geq \sigma\left(t_{1}-t_{0}\right)\left(x_{1}-x^{*}\right)\left(x^{*}-x_{0}\right)=C_{1}>0, \quad t_{1} \leq t \leq T .
$$

Proof of Theorem 4.1. Let $a$ and $b$ belong to $\mathscr{A}$ and let $u$ (resp., $v$ ) be the solution of (1.1) corresponding to $a$ (resp., $b$ ) and $a \neq b$. Without loss of generality, we suppose that either $a(0)>b(0)$ or $a(0)=b(0)$ and $a^{\prime}(0)>b^{\prime}(0)$.

For $a(0)>b(0)$ there exists a $s_{1}, p>0$ such that $a(s)-b(s) \geq p, 0 \leq s \leq s_{1}$, and for $a(0)=b(0)$ and $a^{\prime}(0)>b^{\prime}(0)$ there exists a $s_{1}>0$ such that:

$$
a(s)-b(s) \geq s \frac{a^{\prime}(0)-b^{\prime}(0)}{2}, \quad 0 \leq s \leq s_{1} .
$$

Then, it follows from Proposition 3.2 that there exists a $t_{1}, 0<t_{1} \leq T$, such that:

$$
v(x, t) \leq s_{1}, \quad 0 \leq x \leq 1, \quad 0 \leq t \leq t_{1} .
$$

Now, let $w=u-v$ in $\bar{Q}_{t_{1}}$ with $\left.\left.\left.Q_{t_{1}}=\right] 0,1\right] \times\right] 0, t_{1}[$ and

$$
L=(a \circ u)(x, t) \partial_{x x} \cdot-\partial_{t} \cdot
$$

Then $L[w]+(a(u)-a(v)) \partial_{x x} v=-(a(v)-b(v)) \partial_{x x} v$, in $\bar{Q}_{t_{1}}$.

From the mean value theorem, for any $u$ and $v$ in $[0, M]$ there exists a number $p_{a}(u, v)$ between $u$ and $v$ satisfying $a(u)-a(v)=a^{\prime}\left(p_{a}(u, v)\right)(u-v)$. For $h(x, t)=$ $a^{\prime}\left(p_{a}(u(x, t), v(x, t))\right) \partial_{x x} v(x, t) \geq 0$ in $\bar{Q}_{t_{1}}, w$ is the solution of the problem:

$$
\begin{cases}L[w]+h(x, t) w=-F(x, t), & \text { in } \bar{Q}_{t_{1}}, \\ w(x, 0)=0, & 0 \leq x \leq 1, \\ w(0, t)=0, & 0 \leq t \leq t_{1}, \\ w(1, t)=0, & 0 \leq t \leq t_{1},\end{cases}
$$

where $F(x, t)=[a(v(x, t))-b(v(x, t))] \partial_{x x} v(x, t)$ in $\bar{Q}_{t_{1}}$. Let $z$ be the solution of the problem:

$$
\left\{\begin{aligned}
L[z] & =-F(x, t), & & \text { in } \bar{Q}_{t_{1}}, \\
z(x, 0) & =0, & & 0 \leq x \leq 1, \\
z(0, t) & =0, & & 0 \leq t \leq t_{1}, \\
z(1, t) & =0, & & 0 \leq t \leq t_{1} .
\end{aligned}\right.
$$

From Theorem 1.1 and Corollary 1.1, we deduce that $w \geq z \geq 0$ in $\bar{Q}_{t_{1}}$. Under the stated conditions on $a$ and $b$ we prove that:

$$
w\left(x^{*}, t\right)=u\left(x^{*}, t\right)-v\left(x^{*}, t\right)>0, \quad t^{*} \leq t \leq t_{1}, \quad \text { for some } t^{*}, \quad 0<t^{*}<t_{1} .
$$

1st case. $a(0)>b(0)$ :

In this case $F$ satisfies:

1) $F(x, t) \geq 0$ in $\bar{Q}_{t_{1}}$, and

2) for each $\bar{t}, 0<\bar{t}<t_{1}$, there exists a $C>0$ such that $F(x, t) \geq C, 0 \leq x \leq 1$, $\bar{t} \leq t \leq t_{1}$.

Hence, by Proposition 4.1 for each $t^{*}, 0<t^{*}<t_{1}$, there exists a constant $C_{1}$ such that $z\left(x^{*}, t\right) \geq C_{1}, t^{*} \leq t \leq t_{1}$. Then

$$
w\left(x^{*}, t\right)=u\left(x^{*}, t\right)-v\left(x^{*}, t\right) \geq C_{1}, \quad t^{*} \leq t \leq t_{1} .
$$


2nd case. $a(0)=b(0)$ and $a^{\prime}(0)>b^{\prime}(0)$ :

The second part of Proposition 3.2 implies that $s_{0} \leq v(x, t) \leq s_{1}, t_{0} \leq t \leq t_{1}$, $0 \leq x \leq 1$, for some $t_{0}, 0<t_{0}<t_{1}$ and $s_{0}<s_{1}$. But,

$$
a(s)-b(s) \geq s_{0} \frac{a^{\prime}(0)-b^{\prime}(0)}{2}, \quad s_{0} \leq s \leq s_{1},
$$

and $F$ satisfies in this case:

1) $F(x, t) \geq 0$ in $\bar{Q}_{t_{1}}$, and

2) for each $\bar{t}, t_{0}<\bar{t}<t_{1}$, there exists a $C>0$ such that $F(x, t) \geq C, 0 \leq x \leq 1$, $\bar{t} \leq t \leq t_{1}$.

Now, let $\tilde{z}$ be the solution of the problem:

$$
\begin{cases}L[\tilde{z}]=-F(x, t) & \text { in } \bar{Q}_{t_{0}, t_{1}}, \quad \bar{Q}_{t_{0}, t_{1}}=[0,1] \times\left[t_{0}, t_{1}\right] \\ \tilde{z}\left(x, t_{0}\right)=0, & 0 \leq x \leq 1, \\ \tilde{z}(x, t)=0, & t_{0} \leq t \leq t_{1}, \\ \tilde{z}(x, t)=0, & t_{0} \leq t \leq t_{1} .\end{cases}
$$

It is easy to see that Theorem 1.1 and Proposition 4.1 are still valid if zero is changed to $t_{0}$. Then we have $z \geq \tilde{z}$ in $\bar{Q}_{t_{0}, t_{1}}$ and for each $t^{*}, t_{0}<t^{*}<t_{1}$, there is a constant $C_{1}>0$ such that $z\left(x^{*}, t\right) \geq C_{1}, 0 \leq x \leq 1, t^{*} \leq t \leq t_{1}$. Hence, it follows that $w\left(x^{*}, t\right)=u\left(x^{*}, t\right)-v\left(x^{*}, t\right) \geq C_{1}, t^{*} \leq t \leq t_{1}$, and the proof is complete.

5. Inverse problem. Now, we turn our attention to the hypothesis to be placed on the overspecified data $h$. For $a \in \mathscr{A}, f_{0}, f_{1} \in D_{3,2}, u=u(x, t)$, the solution of (1.1), and $\left.x^{*} \in\right] 0,1\left[\right.$, it follows from Proposition 3.3 that $h=u\left(x^{*}, \cdot\right)$ is in $D_{1} \cap C^{2}[0, T]$, where $D_{1}=\left\{h \in C^{1}[0, T], h(0)=h^{\prime}(0)=0\right.$ and $\left.h^{\prime}(t)>0,0<t \leq T\right\}$.

We formulate the inverse problem as the problem of finding $a$ such that:

$$
\left\{\begin{array}{l}
a \in C^{2}(I) \text { and satisfies }(2.1) \\
u=u(x, t, a) \text { is the solution of }(1.1), \text { and } \\
u\left(x^{*}, \cdot a\right)=h
\end{array}\right.
$$

Lemma 5.1. Let $f_{0}, f_{1} \in D_{3,2}$ and $h \in D_{1} \cap C^{2}[0, T]$. If the inverse problem (5.1) has a solution $a$ in $\mathscr{A}$, then this solution must be unique.

This lemma is just the consequence of Theorem 4.1.

The importance of uniqueness for the inverse problem appears when we consider the numerical determination of the unknown coefficient $a$ from the overspecified data $h$.

Conclusion. In this paper we studied the problem of identifying the unknown coefficient $a$ in a nonlinear diffusion problem from overspecified data measured in the interior of the domain. The identifiability result is obtained for some class of admissible coefficients and appropriate given boundary data. The associated inverse problem is also studied. In a future paper we prove that the identifiability result is more powerful if we change the Dirichlet boundary conditions to Neumann boundary conditions. Finally, we see that the results obtained in this paper are still valid if either $f_{0}=0$ and $f_{1} \neq 0$ or $f_{0} \neq 0$ and $f_{1}=0$. 


\section{REFERENCES}

[1] J. R. Cannon and P. Duchateau, An inverse problem for a nonlinear diffusion equation, SIAM J. Appl. Math. 39, 272-289 (1980)

[2] P. Duchateau, Monotonicity and uniqueness results in identifying an unknown coefficient in a nonlinear diffusion equation, SIAM J. Appl. Math. 41, 310-323 (1981)

[3] P. Duchateau, Boundary behavior and monotonicity estimates for solutions to nonlinear diffusion equations, Rocky Mountain J. Math. 15, 769-786 (1985)

[4] O. A. Ladyzhenskaya, V. A. Solonnikov, and N. N. Uralceva, Linear and quasilinear equations of parabolic type, Amer. Math. Soc., Providence (1968)

[5] O. A. Ladyzhenskaya, Équations quasi-linéaires de types elliptiques et paraboliques, Colloque International $n^{\circ} 117$ sur les équations aux dérivées partielles, Centre National de la Recherche Scientifique, Paris, 1963

[6] M. Protter and H. Weinberger, Maximum principles in differential equations, Prentice-Hall, Englewood Cliffs, N. J., 1967 